Review began 11/22/2021 Review ended 01/17/2022 Published 01/25/2022

\section{() Copyright 2022}

Greb et al. This is an open access article distributed under the terms of the Creative Commons Attribution License CC-BY 4.0. which permits unrestricted use, distribution, and reproduction in any medium, provided the original author and source are credited.

\title{
A Case of Giant Cell Arteritis Presenting After COVID-19 Vaccination: Is It Just a Coincidence?
}

\author{
Christopher S. Greb ${ }^{1}$, Zineb Aouhab ${ }^{2}$, Daniel Sisbarro ${ }^{1}$, Elnaz Panah ${ }^{3}$ \\ 1. Internal Medicine, Loyola University Medical Center, Maywood, USA 2. Rheumatology, Loyola University Medical \\ Center, Maywood, USA 3. Pathology, Loyola University Medical Center, Maywood, USA
}

Corresponding author: Christopher S. Greb, christopher.greb@luhs.org

\begin{abstract}
Giant cell arteritis (GCA) is a large vessel vasculitis with variable presentations, including fevers, myalgias, headache, and jaw claudication. A particularly concerning symptom is transient vision loss, which may become irreversible without prompt recognition and treatment. The pathogenesis of GCA is incompletely understood, but it seems that the innate and adaptive immune systems play a key role in vessel inflammation, remodeling, and occlusion. We present a case of a 79-year-old male who developed GCA two days after he received his second dose of a COVID-19 mRNA vaccine. He presented with headaches, fever, and myalgias. Lab workup revealed elevated inflammatory markers, with C-reactive protein (CRP) $272 \mathrm{mg} / \mathrm{L}$ $(<8.1 \mathrm{mg} / \mathrm{L})$ and erythrocyte sedimentation rate (ESR) $97 \mathrm{~mm} / \mathrm{hr}(0-20 \mathrm{~mm} / \mathrm{hr})$. Imaging of the head, with CT and MRI, was unremarkable. His headache persisted despite supportive treatment, and he developed new, transient blurred vision, which increased suspicion for GCA. He underwent bilateral temporal artery biopsies, which were consistent with GCA. His symptoms resolved quickly with oral prednisone $60 \mathrm{mg}$ daily, and his inflammatory markers returned to normal within a month. A review of the literature revealed several case reports of giant cell arteritis following influenza vaccination. However, no large-scale studies have demonstrated a causal relationship between GCA and immunization. Our case demonstrates the first instance of GCA following a COVID-19 mRNA vaccine. We propose that the upregulated immune response to the vaccine acted as a trigger for GCA in this patient with predisposing factors. While causation cannot be determined based on one case alone, our case demonstrates an opportunity for further research into the relationship between vasculitis and immunizations. Despite this isolated case, the proven benefits of COVID-19 mRNA vaccines significantly outweigh any theoretical risk of immune dysregulation following administration.
\end{abstract}

Categories: Allergy/Immunology, Infectious Disease, Rheumatology

Keywords: vaccine, immunization, covid, covid-19, covid 19, temporal artertitis, gca, giant cell arteritis

\section{Introduction}

Giant cell arteritis (GCA) is a rare, large-vessel vasculitis that affects the aorta and its branches [1]. It has variable presentations, including fever, myalgias, headache, jaw claudication, and transient vision loss. It must be recognized promptly to prevent serious consequences, most notably irreversible vision loss [2]. This can prove difficult, as the triggering events and pathogenesis of GCA are incompletely understood. However, it seems that the innate and adaptive immune systems play a significant role in the amplification of inflammatory pathways that lead to vessel inflammation, remodeling, and occlusion [3]. Several pathogens have been proposed as potential triggers for giant cell arteritis due to their identification in GCA temporal artery biopsies, such as chlamydia pneumonia, parvovirus B19, human herpes viruses, human papillomavirus, and Burkholderia-like bacterium. However, no microorganism or viral pathogen has demonstrated a definitive causal relationship with GCA [4]. In the innate immune system, toll-like receptors activate dendritic cells, which activate $\mathrm{T}$ cells that infiltrate large and medium-sized blood vessels. Activated T cells are regulated by immune checkpoints, such as programmed death-ligand 1 (PD-L1), which may be ineffective in GCA [3]. This idea is supported by the development of giant cell arteritis after checkpoint inhibitor immunotherapy, such as ipilimumab, in some patients with malignant melanoma [3]. In the adaptive immune system, increased T cell production of pro-inflammatory cytokines, such as IL-17A, and decreased activity of regulatory $\mathrm{T}$ cells may contribute to the excessive vessel inflammation in giant cell arteritis [5].

\section{Case Presentation}

We describe a case of a 79-year-old man who presented with nightly headaches for three weeks and transient blurry vision for one week. He reported sharp, debilitating frontal headaches that radiated over the temples bilaterally. The headaches were associated with fever, fatigue, and myalgias. The patient's past medical history included hypertension, hyperlipidemia, atrial fibrillation, hypothyroidism, prostate cancer, and rectal cancer. Symptoms began two days after he received the second dose of a COVID-19 mRNA vaccine. He was seen at an outside hospital emergency department at the onset of symptoms. Infectious workup with chest radiography and urinalysis was unremarkable. He was diagnosed with a mild vaccine reaction and discharged from the emergency department. His symptoms persisted for another week, and he presented to our institution, where he was admitted for further evaluation. Lab workup was remarkable for leukocytosis 


\section{Cureus}

to $16.9 \mathrm{~K} / \mathrm{uL}(3.5-10.5 \mathrm{~K} / \mathrm{uL})$, mild transaminitis, and elevated inflammatory markers, with CRP $272 \mathrm{mg} / \mathrm{L}$ $(<8.1 \mathrm{mg} / \mathrm{L})$ and ESR $97 \mathrm{~mm} / \mathrm{hr}(0-20 \mathrm{~mm})$. Infectious workup, with urinalysis and blood cultures, and head imaging, with CT and MRI, were unremarkable. His headache improved slightly with acetaminophen and IV fluids, and he was discharged after three days. At his follow-up office visit a week later, his headache had become much worse with associated fatigue, scalp tenderness, and three episodes of transient blurred vision in the right and left eye. Each episode resolved after five minutes. He was readmitted due to concern for GCA. On admission, his ophthalmic exam was unremarkable, but he was treated with $60 \mathrm{mg}$ oral prednisone due to high suspicion for GCA. His headache completely resolved less than 12 hours after the administration of steroids. Bilateral temporal artery biopsies were performed the next day, which can be seen in Figures $1 A-1 D$.

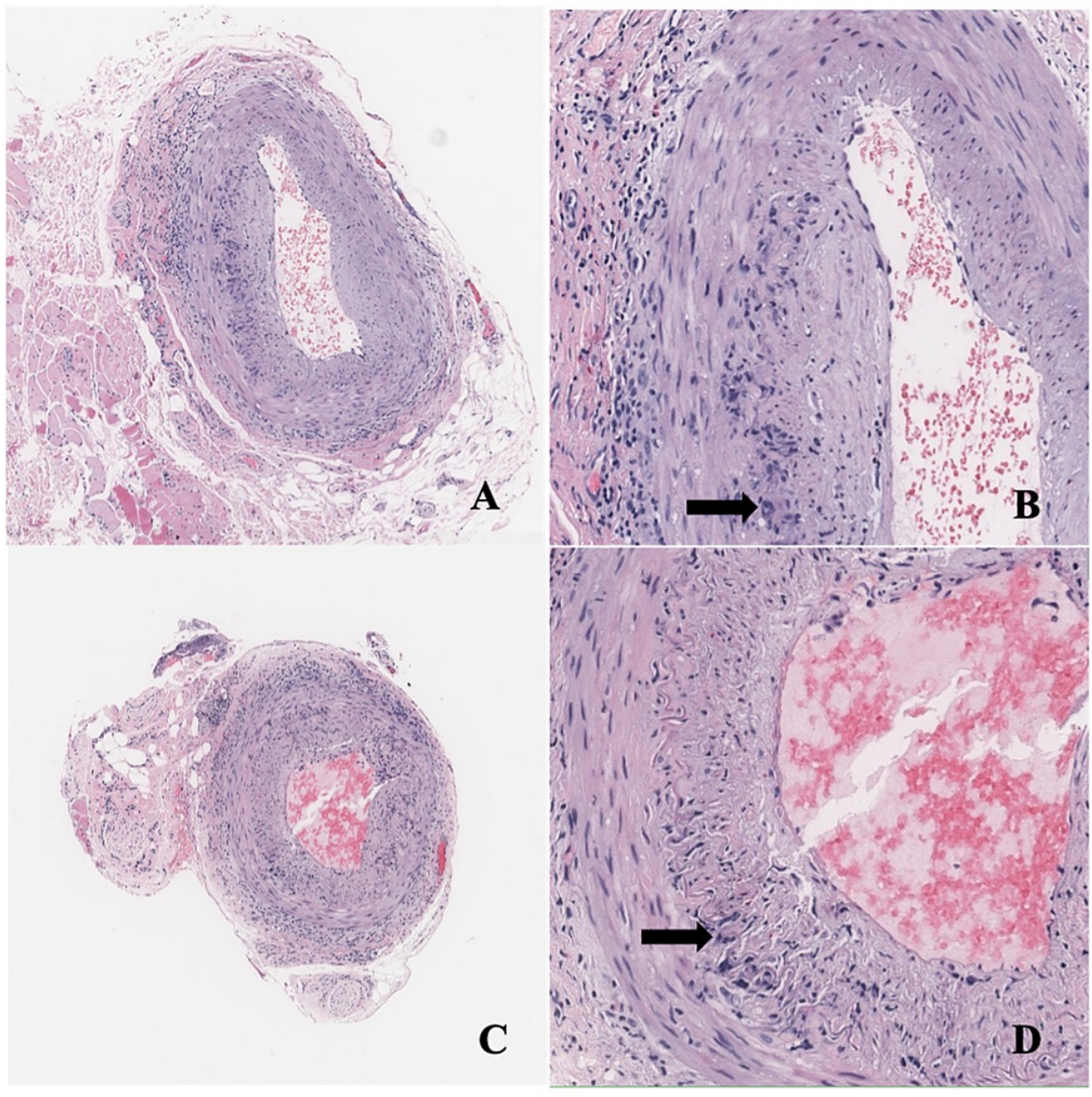

\section{FIGURE 1: Temporal Artery Biopsies}

Right temporal artery (A, B) and left temporal artery (C, D): Medium size muscular artery with intramural inflammatory infiltrates composed of histiocytes, lymphocytes, and eosinophils. Multinucleated giant cells can be identified (arrows). The infiltrate is concentrated at the level of internal elastic lamina and adventitia. Hematoxylin and eosin (H\&E) stain, magnification 50x (A, C), 200x (B, D).

Both biopsies revealed patchy intramural inflammatory infiltrates composed of lymphocytes and rare multinucleated giant cells at the internal lamina and adventitia consistent with a diagnosis of GCA. The patient was discharged on high-dose prednisone (60mg daily), and three weeks later, his CRP and ESR improved to $<1 \mathrm{mg} / \mathrm{L}$ and $2 \mathrm{~mm} / \mathrm{hr}$, respectively. He was tapered to prednisone $50 \mathrm{mg}$ daily by his six-week follow-up appointment without recurrence of symptoms. The timeline of the patient's symptoms and healthcare interactions is illustrated in Figure 2. 


\section{Cureus}

\section{Timeline of Symptoms and Events}

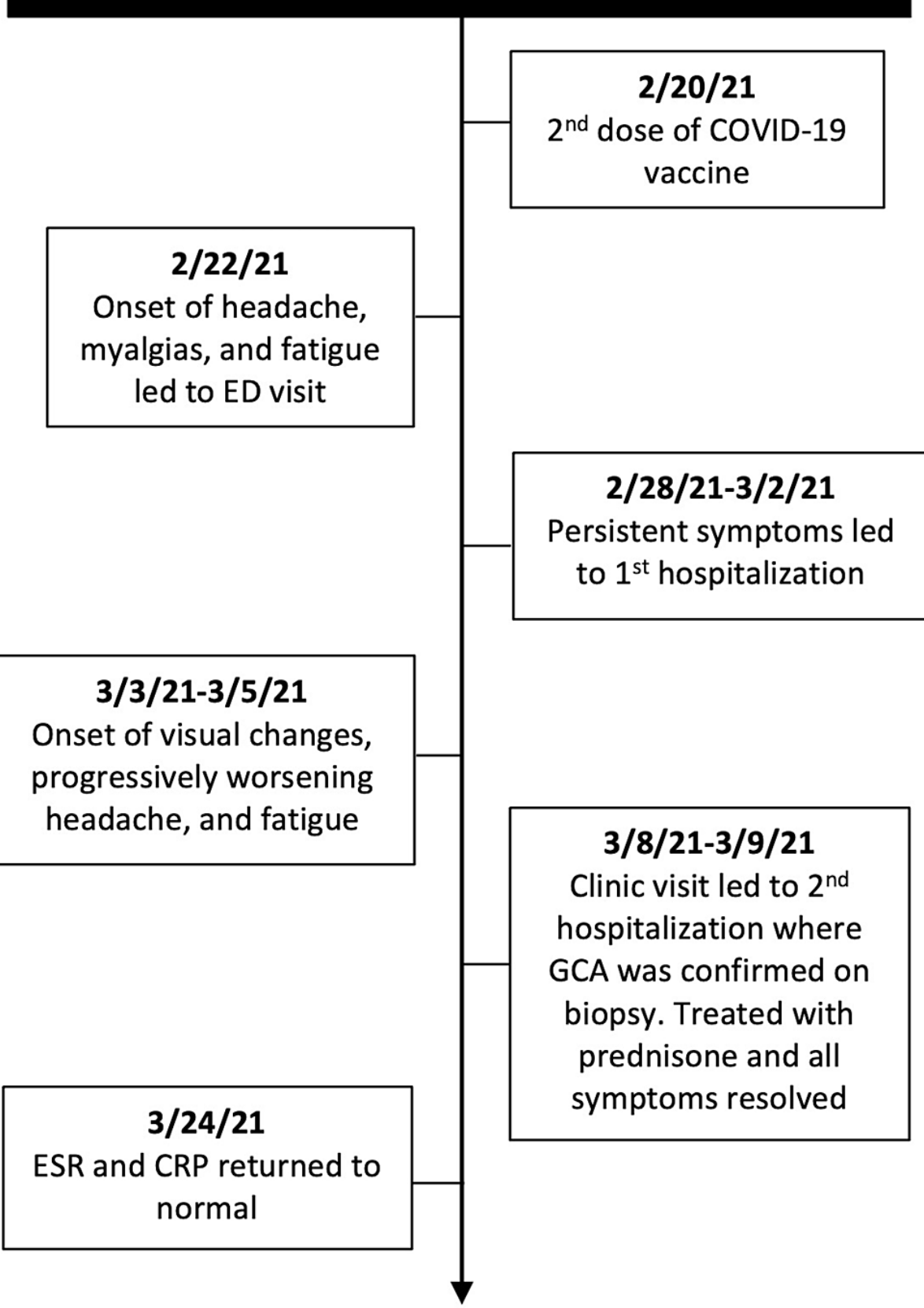

FIGURE 2: Timeline of Symptoms and Events

\section{Discussion}

Concerns have been raised about the relationship between vaccinations and autoimmune disease, including vasculitis. A special focus has been placed on molecular mimicry and the role of adjuvants [6]. These adjuvants increase the innate immune response to foreign antigens, which cause concerns that they may induce reactivity to self-antigens [7]. The COVID-19 mRNA vaccine administered to the patient, in this case, did not contain traditional adjuvants, such as aluminum, seen in other non-mRNA vaccines. However, RNA has intrinsic adjuvant activity, which induces a strong innate immune response [8]. A review of the literature by Guiamares et al. in 2015 revealed 15 cases of GCA following influenza vaccination [9]. Case reports of varying autoimmune diseases after vaccination led Schoenfeld et al. to coin the term autoimmune/inflammatory syndrome induced by adjuvants (ASIA syndrome) to describe a spectrum of immune dysregulation following vaccination [10]. Although a small number of case reports have been published, no epidemiologic studies have shown a definitive increase in autoimmune disease following vaccination. A committee convened in 2015 by the International Life Sciences Institute (ILSI) and Health and 
Environmental Sciences Institute (HESI) conducted an extensive literature review and concluded that there is no compelling evidence supporting the association of vaccine adjuvants and autoimmunity [11]. We reviewed the PubMed and Medline databases by searching case reports with the term "giant cell arteritis" combined with the name of several commonly administered vaccines. No date restriction was placed on the results. The number of cases associated with each vaccine is shown in Table 1.

\begin{tabular}{|l|l|l|}
\hline Vaccine & GCA Cases & References \\
\hline Influenza & 24 & {$[11-19]$} \\
\hline Varicella Zoster & 1 & - \\
\hline COVID-19 & 0 & {$[20]$} \\
\hline TABLE 1: Reported Cases of Giant Cell Arteritis Following Vaccination \\
\hline \hline
\end{tabular}

The review revealed 24 documented cases of GCA after influenza vaccination and one case after varicellazoster vaccination. No cases of giant cell arteritis have been reported following hepatitis A, hepatitis B, tetanus, measles, mumps, rubella, pneumococcal, meningitis, or COVID-19 vaccinations.

\section{Conclusions}

Our case represents the first reported case of giant cell arteritis following vaccination with a COVID-19 mRNA vaccine. We propose that the upregulated immune response to the vaccine acted as a trigger for GCA in this patient with predisposing risk factors. However, causation cannot be reliably determined based solely on the timing of symptoms in this isolated case. Our case demonstrates the opportunity for further largescale studies examining the incidence of GCA in treatment and control groups to better characterize the relationship between GCA and vaccination. Currently, data is limited regarding this relationship. The poor understanding of GCA inciting factors in the literature means clinicians must be diligent when looking for sources of immune hyperreactivity in patients presenting with symptoms suggestive of GCA. While our case suggests opportunities for further research, we strongly endorse continued COVID-19 vaccination, even in elderly patients and those of Northern European ancestry who may be predisposed to developing GCA. During a global pandemic with the highly infectious and lethal COVID-19 virus, the benefits of vaccination far outweigh any theoretical risk of immune dysregulation following administration.

\section{Additional Information \\ Disclosures}

Human subjects: All authors have confirmed that this study did not involve human participants or tissue. Conflicts of interest: In compliance with the ICMJE uniform disclosure form, all authors declare the following: Payment/services info: All authors have declared that no financial support was received from any organization for the submitted work. Financial relationships: All authors have declared that they have no financial relationships at present or within the previous three years with any organizations that might have an interest in the submitted work. Other relationships: All authors have declared that there are no other relationships or activities that could appear to have influenced the submitted work.

\section{References}

1. Crowson CS, Matteson EL, Myasoedova E, et al.: The lifetime risk of adult-onset rheumatoid arthritis and other inflammatory autoimmune rheumatic diseases. Arthritis Rheum. 2011, 63:633-9. 10.1002/art.30155

2. Danesh-Meyer H, Savino PJ, Gamble GG: Poor prognosis of visual outcome after visual loss from giant cell arteritis. Ophthalmology. 2005, 112:1098-103. 10.1016/j.ophtha.2005.01.036

3. Terrades-Garcia N, Cid MC: Pathogenesis of giant-cell arteritis: how targeted therapies are influencing our understanding of the mechanisms involved. Rheumatology (Oxford). 2018, 57:ii51-62. 10.1093/rheumatology/kex423

4. van Timmeren MM, Heeringa P, Kallenberg CG: Infectious triggers for vasculitis. Curr Opin Rheumatol. 2014, 26:416-23. 10.1097/BOR.0000000000000068

5. Espígol-Frigolé G, Corbera-Bellalta M, Planas-Rigol E, et al.: Increased IL-17A expression in temporal artery lesions is a predictor of sustained response to glucocorticoid treatment in patients with giant-cell arteritis. Ann Rheum Dis. 2013, 72:1481-7. 10.1136/annrheumdis-2012-201836

6. Segal Y, Shoenfeld Y: Vaccine-induced autoimmunity: the role of molecular mimicry and immune crossreaction. Cell Mol Immunol. 2018, 15:586-94. 10.1038/cmi.2017.151

7. Perricone C, Colafrancesco S, Mazor RD, Soriano A, Agmon-Levin N, Shoenfeld Y: Autoimmune/inflammatory syndrome induced by adjuvants (ASIA) 2013: unveiling the pathogenic, clinical and diagnostic aspects. J Autoimmun. 2013, 47:1-16. 10.1016/j.jaut.2013.10.004

8. Mulligan MJ, Lyke KE, Kitchin N, et al.: Phase I/II study of COVID-19 RNA vaccine BNT162b1 in adults . Nature. 2020, 586:589-93. 10.1038/s41586-020-2639-4 


\section{Cureus}

9. Guimarães LE, Baker B, Perricone C, Shoenfeld Y: Vaccines, adjuvants and autoimmunity. Pharmacol Res. 2015, 100:190-209. 10.1016/j.phrs.2015.08.003

10. Shoenfeld Y, Agmon-Levin N: 'ASIA' - autoimmune/inflammatory syndrome induced by adjuvants . J Autoimmun. 2011, 36:4-8. 10.1016/j.jaut.2010.07.003

11. van der Laan JW, Gould S, Tanir JY: Safety of vaccine adjuvants: focus on autoimmunity . Vaccine. 2015, 33:1507-14. 10.1016/j.vaccine.2015.01.073

12. Wada M, Asai J, Asai A, Nakai D, Kishimoto S, Katoh N: Giant cell arteritis with polymyalgia rheumatica associated with influenza vaccination. J Dermatol. 2011, 38:1099-101. 10.1111/j.1346-8138.2011.01277.x

13. Konishi M, Koarada S, Yamaguchi K, et al.: A case of microscopic polyangiitis and giant cell arteritis after influenza vaccination. Jap J Cln Immu. 2011, 34:154-61. 10.2177/jsci.34.154

14. Perez C, Loza E, Tinture T: Giant cell arteritis after influenza vaccination. Arch Intern Med. 2000, 160:2677.

15. Soriano A, Verrecchia E, Marinaro A, Giovinale M, Fonnesu C, Landolfi R, Manna R: Giant cell arteritis and polymyalgia rheumatica after influenza vaccination: report of 10 cases and review of the literature. Lupus. 2012, 21:153-7. 10.1177/0961203311430222

16. Finsterer J, Artner C, Kladosek A, Kalchmayr R, Redtenbacher S: Cavernous sinus syndrome due to vaccination-induced giant cell arteritis. Arch Intern Med. 2001, 161:1008-9. 10.1001/archinte.161.7.1008-a

17. Ghose MK, Shensa S, Lerner PI: Arteritis of the aged (giant cell arteritis) and fever of unexplained origin . Am J Med. 1976, 60:429-436. 10.1016/0002-9343(76)90760-9

18. Liozon E, Parreau S, Filloux M, et al.: Giant cell arteritis or polymyalgia rheumatica after influenza vaccination: a study of 12 patients and a literature review. Autoimmun Rev. 2021, 20:102732. 10.1016/i.autrev.2020.102732

19. Pou MA, Diaz-Torne C, Vidal S, Corchero C, Narvaez J, Nolla JM, Diaz-Lopez C: Development of autoimmune diseases after vaccination. J Clin Rheumatol. 2008, 14:243-4. 10.1097/RHU.0b013e318181b496

20. Nichani P, Micieli JA: Granuloma annulare, scalp necrosis, and ischemic optic neuropathy from giant cell arteritis after Varicella-Zoster virus vaccination. J Neuroophthalmol. 2021, 41:e145-8.

10.1097/WNO.0000000000000947 